\title{
Fields radiated by a nanoemitter in a graphene sheet
}

\author{
A. Yu. Nikitin,,${ }^{1,2}{ }^{*}$ F. Guinea ${ }^{3}$ F. J. Garcia-Vidal, ${ }^{4}$ and L. Martin-Moreno ${ }^{1, \dagger}$ \\ ${ }^{1}$ Instituto de Ciencia de Materiales de Aragón and Departamento de Física de la Materia Condensada, CSIC-Universidad de Zaragoza, \\ E-50009 Zaragoza, Spain \\ ${ }^{2}$ A. Ya. Usikov Institute for Radiophysics and Electronics, Ukrainian Academy of Sciences, 12 Acad. Proskura Str., 61085 Kharkov, Ukraine \\ ${ }^{3}$ Instituto de Ciencia de Materiales de Madrid, CSIC, Cantoblanco, E-28049 Madrid, Spain \\ ${ }^{4}$ Departamento de Física Teórica de la Materia Condensada, Universidad Autónoma de Madrid, E-28049 Madrid, Spain
}

(Received 3 November 2011; published 18 November 2011)

\begin{abstract}
The extraordinary properties of graphene make it a very promising material for optoelectronics. However, basic attributes of the electromagnetic field in graphene are still unexplored. Here we report on the in-plane fields radiated by a nanoemitter lying on a graphene sheet in terahertz regime, which present a rich dependence on frequency, distance to the source, and orientation of the dipole moment. The field pattern is mainly composed of a core region, dominated by surface plasmons, where the electric field can be several orders of magnitude larger than in vacuum, and an outer region where the field is virtually the same as what it would be in vacuum.
\end{abstract}

DOI: 10.1103/PhysRevB.84.195446

PACS number(s): 73.20.Mf, 41.20.Jb, 42.25.Bs, 42.79.Ag

\section{INTRODUCTION}

Graphene has attracted recently a great deal of attention due to its amazing electronic properties. ${ }^{1,2}$ On top of fundamental issues, such as being the thinnest possible two-dimensional electron gas (2DEG) and possessing charged massless quasiparticles, graphene presents very interesting material properties for its use in electronics. Especially noteworthy are the possibility of controlling its chemical potential $\mu$ (and thus the conductivity $\sigma$ ) through gate voltages and its high mobility at room temperature. Also, the optical properties of graphene are exceptional, ${ }^{3,4}$ as they allow the visualization of a material that is just one atom thick, and even allow differentiation of regions with different numbers of atomic layers. ${ }^{5,6}$ Remarkably, even one-atom-thick graphene can bind electromagnetic (EM) modes, despite being almost transparent. These surface EM modes are surface plasmon polaritons (SPPs) with a transverse-magnetic (TM) polarization ${ }^{7-10}$ when $\operatorname{Im} \sigma>0$, which occurs below a critical frequency $\omega_{0}$ that depends on $\mu$. For frequencies above $\omega_{0}, \operatorname{Im} \sigma<0$ and graphene supports transverse-electric (TE) bound EM modes, ${ }^{11}$ which are reminiscent of the guided modes in a dielectric film. The existence of SPPs in graphene, with their similarities to those supported by metal surfaces, brings to the fore the possibility of using graphene for many of the functionalities sought within the field of plasmonics. For instance, graphene could be used to built terahertz (THz) switches, ${ }^{12}$ and a graphene sheet with a spatially nonuniform conductivity is a promising platform for both $\mathrm{THz}$ flatland metamaterials and transformation optics. $^{13}$

For all these optical functionalities, an essential requirement is the efficient excitation of the surface EM modes. In this work we analyze how these bound modes are excited by an emitter of deep subwavelength dimensions (quantum dots, molecules, dielectric or metallic protuberances, etc.) in graphene. Additionally, we study the EM-field pattern at the graphene layer, which is an essential ingredient for the understanding of EM properties of nanostructures placed on a graphene sheet and the effective EM interaction between them.

\section{DESCRIPTION OF THE SYSTEM UNDER STUDY}

We consider a point emitter (placed at the origin, with dipole moment p) lying on a free-standing graphene sheet (covering the plane $z=0$ ). Graphene is represented by its in-plane complex conductivity $\sigma$, which is a function of both frequency $\omega$ and material properties, such as $\mu$ and temperature $T$. The conductivity $\sigma$ is taken from calculations based on the random-phase approximation, ${ }^{14-16}$ and is represented in the inset of Fig. 1(b). In this work, we consider $T=300 \mathrm{~K}$ and $\mu=0.2 \mathrm{eV}(48 \mathrm{THz})$, which are typical experimental values. Nevertheless, we have checked that all results presented in this paper are qualitatively valid for other values of $\mu$ (provided $\omega$ is scaled in the same manner) and $T$, and under the presence of a dielectric substrate (as long as the substrate does not support optical resonances in the frequency range of interest).

\section{MATHEMATICAL FORMULATION}

The electric field radiated by the dipole can be written as $\mathbf{E}(\mathbf{r})=\hat{G}(\mathbf{r}) \mathbf{p}$, where $\hat{G}(\mathbf{r})$ is the Green's dyadic of the problem. We express distances $R$ in dimensionless units as $r=k_{\omega} R=2 \pi R / \lambda$, with $k_{\omega}=\omega / c, c$ is the speed of light, and $\lambda$ is the wavelength of the EM field. Similarly, we define dimensionless quantities for wave vectors (through $q=k / k_{\omega}$ ), conductivity $(\alpha=2 \pi \sigma / c)$, and frequency $(\Omega=\hbar \omega / \mu)$. Due to the symmetry of the problem, it is convenient to work in cylindrical coordinates, $\mathbf{r}=(r, \theta, z)$ (see Fig. 1). Following standard techniques in electromagnetism (see for instance Ref. 17), $\hat{G}$ can be represented in terms of Sommerfeld integrals:

$$
\hat{G}_{i j}=\frac{i k_{\omega}}{8 \pi} \sum_{\tau=\mathrm{TE}, \mathrm{TM}} \int_{0}^{\infty} d q \hat{D}_{i j}^{\tau}(q) \hat{J}_{i j}^{\tau}(q r),
$$

where $i$ and $j$ are $r, \theta$, or $z, \hat{J}_{i j}^{\tau}(q r)$ are combinations of Bessel functions, and $D_{i j}^{\tau}$ are the components of the angular spectrum dyadic (see Appendixes B and $\mathrm{C}$ for the derivation and explicit expressions). The locations of poles of $D_{i j}^{\tau}$ in complex $q$ space, $q_{p}$, provide the spectral region of existence of SPPs 

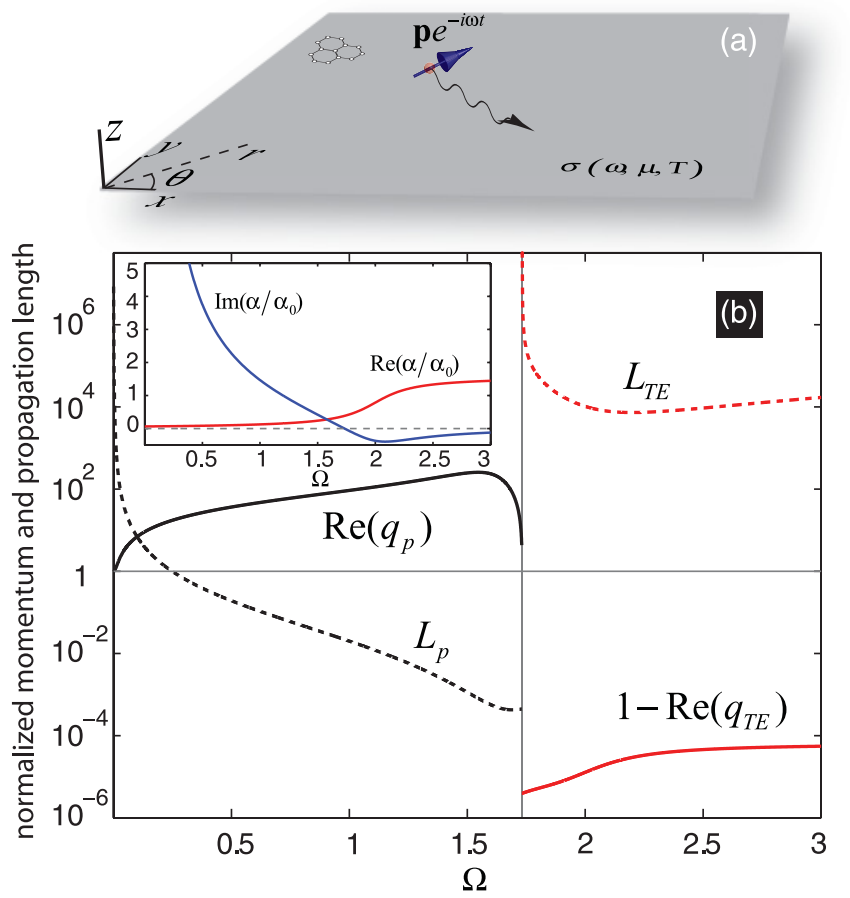

FIG. 1. (Color online) (a) The geometry of the studied system. (b) Dispersion relation $q(\Omega)$ (continuous curves) and propagation length (discontinuous curves) of SPPs and TE bound modes, where $q$ is the wave vector in units of $\omega / c$ and $\Omega=\hbar \omega / \mu$. These magnitudes are obtained for the case in which $\mu=0.2 \mathrm{eV}(48 \mathrm{THz})$ and $T=$ $300 \mathrm{~K}$. The inset shows $\alpha=2 \pi \sigma / c$ in units of the fine-structure constant $\alpha_{0} \simeq 1 / 137$.

and bounded TE modes, their corresponding wave vectors $\operatorname{Re}\left(q_{p}\right)$, and their propagation lengths $L_{p}=\lambda /\left[2 \pi \operatorname{Im}\left(q_{p}\right)\right]$, which are rendered in Fig. 1(b). The nonzero components of $D_{i j}^{\tau}$ are represented along the real $q$ axis in Fig. 2, for both the graphene sheet (main figure, for $\Omega=0.02$ ) and free space (inset). For this frequency, SPPs are supported by the sheet, as clearly seen in the presence of a peak in the TM components of the angular spectrum of graphene at $\operatorname{Re}\left(q_{p}\right) \equiv k_{p} / k_{\omega} \approx 1.7$. In principle, $\hat{G}_{i j}(r)$ must be computed numerically. However, some heuristic properties can be extracted from the form of Eq. (1) and with the visual help of Fig. 2. Equation (1) shows that the EM field originates from the interference of a continuum of oscillatory functions $J_{i j}^{\tau}(q r)$ (which are given by the symmetry of the problem), each of them being launched with an amplitude $D_{i j}^{\tau}$ (which depends on the material properties). For large distances, the oscillatory functions oscillate very quickly with $q$, and the contributions to the integral of "smooth" (in a scale $\sim 1 / r$ ) parts of the angular spectrum cancel out. Thus, only the sharpest spectral features in $D_{i j}^{\tau}$ are relevant at long distances from the source. These can be divided in three classes: (i) square-root singularities, which appear for some components at the light cone $(q=1)$ and lead to a $1 / r$ dependence of the field at large distances, (ii) poles associated to bound EM modes which decay exponentially with distance both in the normal and in-plane directions, and (iii) kinks (points where the derivative of the angular spectrum is discontinuous) appearing for some components at $q=1$ which lead to $1 / r^{2}$ decays. The expressions for these

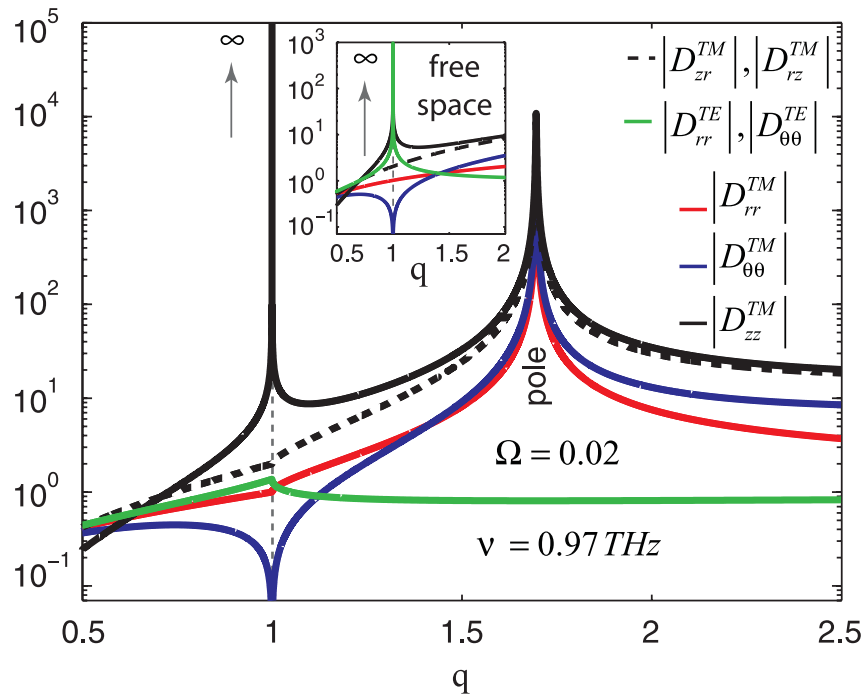

FIG. 2. (Color online) The different components of the angularspectrum dyadic $\hat{D}$ as a function of the normalized in-plane wave vector $q=c k / \omega$. The main figure corresponds to the graphene sheet (for the case $T=300 \mathrm{~K}$ and $\mu=0.2 \mathrm{eV}$ ) while the inset is for vacuum. In both cases $\Omega=0.02$, which corresponds to $v \approx 1 \mathrm{THz}$ $(\lambda=0.3 \mathrm{~mm})$ for the $\mu$ considered.

contributions are given in Appendix C. As we will show later, all these spectral features show up in the EM field in different spatial regions.

In what follows we present results for the electric field at the graphene sheet radiated by a nanoemitter as a function of frequency $\omega$. Let us analyze separately the emission by considering the different components of the dipole.

\section{PERPENDICULAR DIPOLE, $p=p \mathrm{e}_{z}$}

Figure 3 presents the spatial dependence of the electric field at the graphene sheet. Due to the symmetry of the problem the field does not depend on the polar angle $\theta$, as illustrated in Fig. 3(a). Figures 3(b)-3(d) render the results for four different frequencies, each one being representative of a different regime. In each case, the exact electric field ( $E$, red lines) is plotted together with the SPP contribution $\left(E^{\mathrm{SPP}}\right.$, black lines) and, for reference, the electric field that the dipole would radiate in free space ( $E^{\mathrm{FS}}$, blue lines).

Figure 3(b) renders the results for $\Omega=0.02$, which is a representative case of the small- frequency regime $\hbar \omega \ll \mu$. In this case, $k_{p} \approx k_{\omega}$ and $L_{p} \gg \lambda$. As the figure shows, the exact field virtually coincides with the SPP contribution even at distances much smaller than the wavelength. At larger distances the exponentially decaying SPP dominates until it is superseded by an algebraically decaying field, which turns out to be well approximated by the free-space radiation [see inset to Fig 3(b)]. The crossover distance $R_{c}$ typically occurs at $R_{c} \sim 10 L_{p}$ which, for this frequency regime, may be hundreds or even thousands of wavelengths. The field oscillates with a similar period before and after $R_{c}$, presenting an interference pattern at distances close to $R_{c}$, where SPP and free-space (FS) fields are of the same order. 

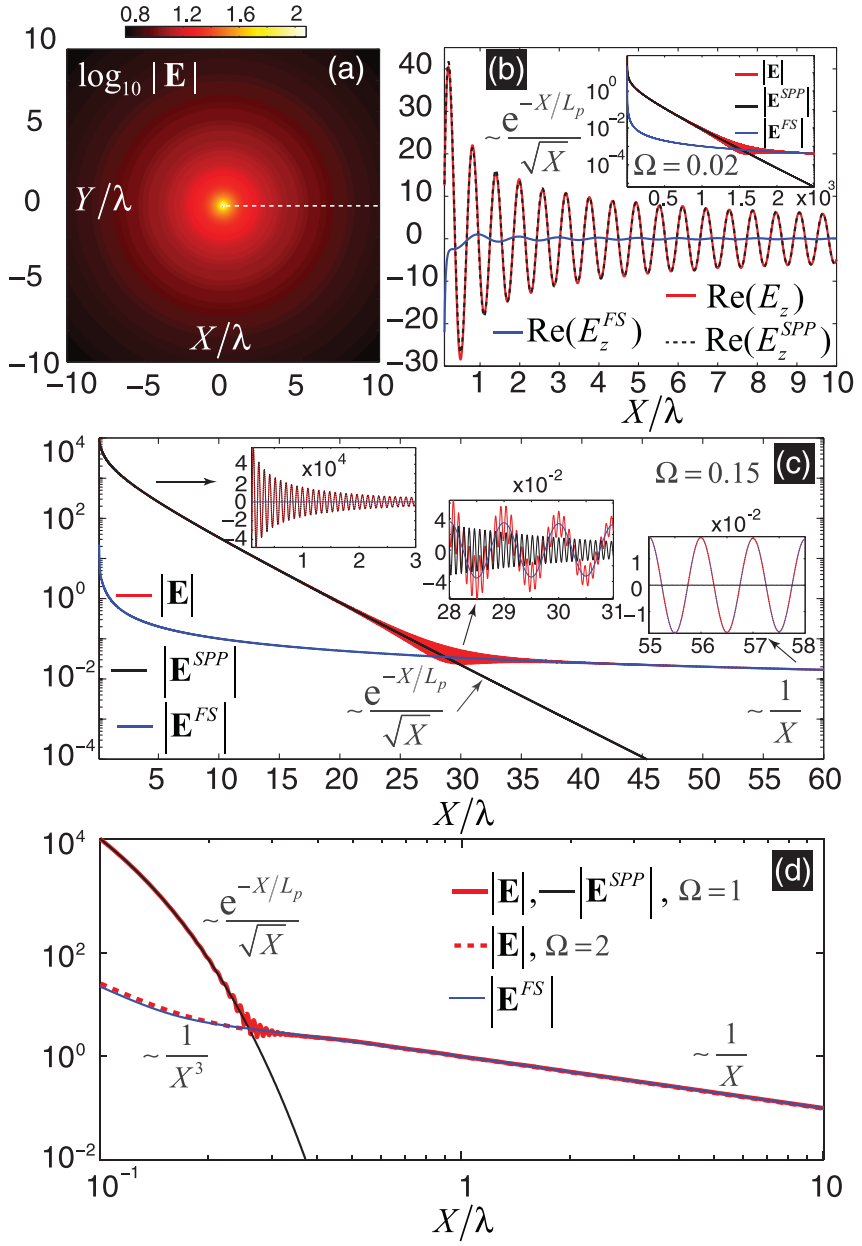

FIG. 3. (Color online) Spatial dependencies of the electric field radiated by a dipole perpendicular to the graphene sheet, $\mathbf{p}=p \mathbf{e}_{z}$. In (a) the field color map for $\log _{10}(|\mathbf{E}|)$ is shown for $\Omega=0.02$. The white discontinuous line represents the section along which all the 1D field dependencies are shown. In (b)-(d), and their insets, both $\log _{10}(|\mathbf{E}|)$ and $\operatorname{Re}\left(E_{z}\right)$ are plotted as functions of distance for different $\Omega$. In all the panels together with the full field, both surface plasmon and free-space contributions are shown. All fields are normalized to $k_{\omega} p / 2$, which is the largest value of the field radiated by a dipole moment in free space at distance $r=\lambda$.

Figure 3(c) is representative of an intermediate frequency range $(\Omega=0.15)$, in which $k_{p} \gg k_{\omega}$ and $L_{p}$ is a few times the wavelength. In this range the field can also be well approximated by the sum of two terms: the SPP (which dominates at short distances) and a "free-space" wave (prevailing at long distances). However, now the spatial periods of the EM field at $R<R_{c}$ and $R>R_{c}$ are vastly different [see insets to Fig. 3(c)]. For higher frequencies, $L_{p}<\lambda_{p}<\lambda$ and the SPP is an overdamped wave $(1.2<\Omega<1.6$ for the temperature and chemical potential considered in this paper; see Fig. 1). Still the field at the graphene sheet can be represented by an SPP at $R<R_{c}$ and as free-space radiation for larger distances [see Fig. 3(d) for $\Omega=1$ ). However, in this case $R_{c}$ is in the subwavelength regime, and in fact it vanishes as $\Omega \rightarrow \Omega_{0}\left(\Omega_{0}=1.6\right.$ for the material parameters considered in this paper). Nevertheless, note that the huge amplification of the $E$ field in the vicinity of the emitter is still maintained thanks to the SPP excitation. For frequencies $\Omega>\Omega_{0}$, the graphene layer does not support SPPs but it does support TE surface waves. However, as represented in Fig. 3(d) for $\Omega=2$, the fields at the graphene sheet are very approximately the same as if the graphene layer were not present. The TE field is so weakly bounded that it virtually does not couple to the point emitter.

An important message that can be extracted from Fig. 3 is that, in all cases where SPPs are supported by the graphene sheet, the intensity of the field at distances from the source smaller than $R_{c}$ is orders or magnitude larger than in free space. This is related to the strong Purcell factor induced by graphene. ${ }^{18}$ Our results show that the enhancement into the plasmon channel can be as large as $10^{5}-10^{6}$. This amplification, combined with the tunability of the chemical potential (and thus of $\Omega$ ) through doping levels and/or external gates, may have important practical applications. For instance, whether or not a subwavelength region close to the emitter
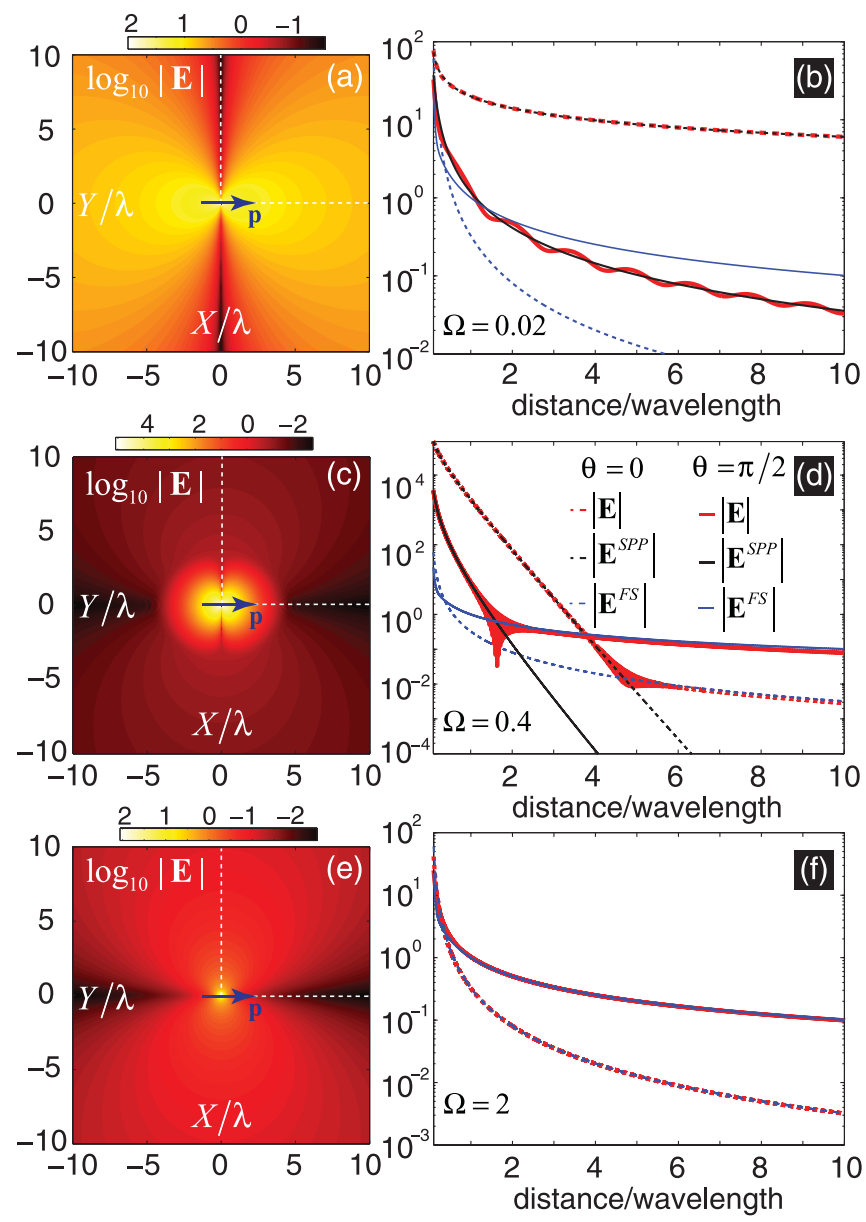

FIG. 4. (Color online) The spatial dependencies of the electric field radiated by a dipole parallel to the graphene sheet $\mathbf{p}=p \mathbf{e}_{x}$ for different $\Omega: \Omega=0.02(v=1 \mathrm{THz}, \lambda=0.3 \mathrm{~mm}), \Omega=0.4(v=$ $19.4 \mathrm{THz}, \lambda=15.5 \mu \mathrm{m})$, and $\Omega=2(v=97 \mathrm{THz}, \lambda=3.1 \mu \mathrm{m})$. In (a), (c), and (e) the field color maps for $\log _{10}(|\mathbf{E}|)$ are shown. The white discontinuous lines represent the directions along which all the 1D field dependencies are shown in (b), (d), and (f). In all the panels together with the full field, both surface plasmon and free-space contributions are shown. The normalization of the fields is the same as in Fig. 3. 
is strongly illuminated (and the extent of this region) can be externally controlled by DC voltages.

\section{PARALLEL DIPOLE, $\mathrm{p}=p \mathrm{e}_{x}$}

Let us consider now a dipole parallel to the graphene sheet, in a direction that we take to be the $x$ direction (i.e., $\theta=0)$. The electric field at the graphene sheet is $\mathbf{E}(r, \theta)=$ $\left(G_{r r}(r) \cos \theta, G_{\theta \theta}(r) \sin \theta, G_{z r}(r) \cos \theta\right)^{T} p$, where $T$ stands for transposition.

In contrast to the case of the perpendicular dipole, the electric field radiated by the parallel dipole is strongly angular dependent, and this dependency drastically changes with $\Omega$; see the color maps in Fig. 4. Overall, the field pattern follows the rules previously discussed for the vertical dipole: (i) For $\Omega<\Omega_{0}$, SPPs are sustained and dominate the field in an inner core region, with a size related to the SPP decay length. Outside this core region, the field at the graphene sheet is well approximated by the one in free space. (ii) For $\Omega>\Omega_{0}$, the core region has vanished and the field is everywhere practically the same as in free space. However, in the case of the in-plane dipole there are two main differences. The first one is that, strictly speaking, the long-distance limit of the in-plane field is not the transversal free-space field. Instead, the asymptotic field arises from the kink in the angular spectrum (see Fig. 2), and is reminiscent of the Norton wave found in the studies of radio transmission at the Earth's surface ${ }^{19}$ and of radiation by holes in metals. ${ }^{20}$ This asymptotic field decays as $r^{-2}$ and is predominantly TM polarized. Notice however that the small value of the field in the region where NW dominates most probably prevents its experimental detection. Our calculations show that the Norton wave in graphene is predominantly TM polarized. Figure 5 renders a "phase diagram" showing the main character of the field as a function of both frequency and distance to the source. The second difference is related to the polarization of the inner core and outer region. As SPPs have a longitudinal field component [thus vanishing at $\theta=\pi / 2$; see Fig. 4(a)] and FS radiation is transversal [vanishing at $\theta=0$; see Fig. 4(e)], the polarization patterns of the inner core and

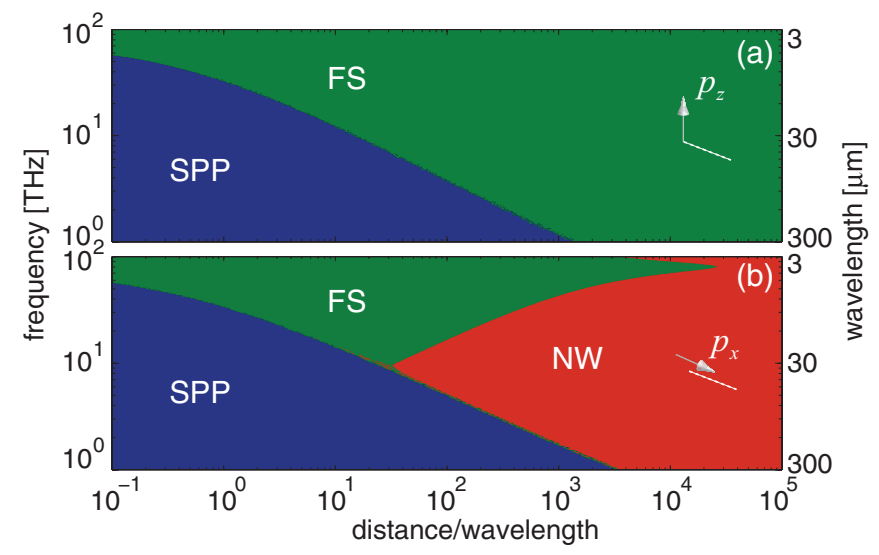

FIG. 5. (Color online) Phase diagram showing the main character of the field (SPP, FS, or NW) at the graphene sheet as a function of source frequency and distance to the source. Panel (a) is for a dipole perpendicular to the sheet, while panel (b) is for a parallel one. In both cases $T=300 \mathrm{~K}$ and $\mu=0.2 \mathrm{eV}$. the outer region are rotated by $\pi / 2$, as illustrated in Fig. 4(c). Correspondingly, for $\Omega<\Omega_{0}$, the inner core region extends further away at $\theta=0$ than at $\theta=\pi / 2$ [see Fig. 4(d)].

\section{CONCLUSIONS}

Our results show that the field patterns are drastically different for different frequency ranges and orientations of the dipole moment. The behavior of the fields is related to distinct peculiarities of the density of electromagnetic states in graphene. The field pattern is mainly composed of a core region dominated by surface plasmons, where the electric field can be several orders of magnitude larger than in vacuum, and an outer region where the field is virtually the same as what it would be in vacuum. Importantly, the core-region size can be controlled thorough external gates, which opens up many interesting applications in, for instance, surface optics, spectroscopy, and the tailoring of the interaction between quantum objects.

\section{ACKNOWLEDGMENTS}

The authors acknowledge support from the Spanish Ministry of Science and Innovation under Grants No. MAT200906609-C02 and No. CSD2007-046-NanoLight.es. A.Y.N. acknowledges Juan de la Cierva Grant No. JCI-2008-3123.

\section{APPENDIX A: THE MODEL FOR THE CONDUCTIVITY OF GRAPHENE}

The conductivity of graphene has been computed within the random-phase approximation. ${ }^{14-16}$ In terms of the chemical potential $\mu$ and the temperature $T$, the conductivity can be expressed as

$$
\sigma=\sigma_{\text {intra }}+\sigma_{\text {inter }}
$$

where, for relaxation times much larger that $\omega^{-1}$, the intraband and interband contributions are

$$
\begin{aligned}
\sigma_{\text {intra }}= & \frac{2 i e^{2} t}{\hbar \pi \Omega} \ln \left[2 \cosh \left(\frac{1}{2 t}\right)\right], \\
\sigma_{\text {inter }}= & \frac{e^{2}}{4 \hbar}\left[\frac{1}{2}+\frac{1}{\pi} \arctan \left(\frac{\Omega-2}{2 t}\right)\right. \\
& \left.-\frac{i}{2 \pi} \ln \frac{(\Omega+2)^{2}}{(\Omega-2)^{2}+(2 t)^{2}}\right] .
\end{aligned}
$$

In these expressions $\Omega=\hbar \omega / \mu$ and $t=T / \mu$, with $T$ expressed in units of energy.

A finite relaxation time $\tau$ can customarily taken into account by substituting $\omega$ with $\omega+\imath \tau^{-1}$ in $\sigma_{\text {intra }}$.

\section{APPENDIX B: THE ELECTROMAGNETIC GREEN'S DYADIC FOR A 2D ELECTRON GAS}

Throughout this Appendix, we express coordinates in the in-plane $(\mathbf{R})$ and normal $(Z)$ directions to the graphene sheet in dimensionless units as $\mathbf{r}=k_{\omega} \mathbf{R}=2 \pi \mathbf{R} / \lambda$ and $z=k_{\omega} Z$.

The electric field $\mathbf{E}(\mathbf{r}, z)$ emitted by an electric dipole, with dipole moment $\mathbf{p}\left(z^{\prime}\right)$ and placed at the point $\left(\mathbf{r}^{\prime}=0, z^{\prime}\right)$, is given 
through the Green's dyadic (GD) $\hat{G}\left(\mathbf{r}, z, z^{\prime}\right)=\hat{G}\left(\mathbf{r}, z ; \mathbf{r}^{\prime}=\right.$ $\left.0, z^{\prime}\right)$ by the relation

$$
\mathbf{E}(\mathbf{r}, z)=\hat{G}\left(\mathbf{r}, z ; z^{\prime}\right) \mathbf{p}\left(z^{\prime}\right) .
$$

General form of the Green's dyadic under the presence of a $2 \mathrm{D}$ electron gas. The GD can be expressed (see for instance Refs. 17 and 21) in terms of the electromagnetic modes in free space, $\mathbf{u}_{\mathbf{q} \tau} e^{i \mathbf{q r}+i q_{z} z}$, characterized by their in-plane momentum $\mathbf{q}=\mathbf{k} / k_{\omega}$ and polarization $\left.\tau=\mathrm{TE}, \mathrm{TM}\right)$ :

$$
\begin{aligned}
& \hat{G}\left(\mathbf{r}, z ; z^{\prime}\right)=\hat{G}_{0}\left(\mathbf{r}, z ; z^{\prime}\right)+\hat{G}_{R}\left(\mathbf{r}, z ; z^{\prime}\right), \quad z^{\prime}<0, \quad z<0, \\
& \hat{G}\left(\mathbf{r}, z ; z^{\prime}\right)=\hat{G}_{T}\left(\mathbf{r}, z ; z^{\prime}\right), \quad z^{\prime}<0, \quad z>0,
\end{aligned}
$$

with $\hat{G}_{0}$ being the GD in free space,

$$
\hat{G}_{0}\left(\mathbf{r}, z ; z^{\prime}\right)=\sum_{\tau} \int \frac{d \mathbf{q}}{2 q_{z}} \mathbf{u}_{\mathbf{q} \tau}^{ \pm} \mathbf{u}_{\mathbf{q} \tau}^{ \pm T} e^{i \mathbf{q r}+i q_{z}\left|z-z^{\prime}\right|},
$$

and $\hat{G}_{R}, \hat{G}_{T}$ being the contributions due to the reflection and transmission in our 2D system,

$$
\begin{aligned}
& \hat{G}_{r}\left(\mathbf{r}, z ; z^{\prime}\right)=\sum_{\tau} \int \frac{d \mathbf{q}}{2 q_{z}} r_{q}^{\tau} \mathbf{u}_{\mathbf{q} \tau}^{+} \mathbf{u}_{\mathbf{q} \tau}^{-T} e^{i \mathbf{q r}-i q_{z}\left(z+z^{\prime}\right)}, \\
& \hat{G}_{t}\left(\mathbf{r}, z ; z^{\prime}\right)=\sum_{\tau} \int \frac{d \mathbf{q}}{2 q_{z}} t_{q}^{\tau} \mathbf{u}_{\mathbf{q} \tau}^{+} \mathbf{u}_{\mathbf{q} \tau}^{+T} e^{i \mathbf{q} \mathbf{r}+i q_{z}\left(z-z^{\prime}\right)} .
\end{aligned}
$$

In these expressions $T$ stands for transposition, "+" corresponds to the regions where $z>z^{\prime}$ while "-_" is for $z<z^{\prime}$, and the unitary vectors characterizing the polarization of each mode are

$$
\mathbf{u}_{\mathbf{q T E}}^{ \pm}=\frac{1}{q}\left(\begin{array}{c}
-q_{y} \\
q_{x} \\
0
\end{array}\right), \quad \mathbf{u}_{\mathbf{q T M}}^{ \pm}=\frac{q_{z}}{q}\left(\begin{array}{c}
q_{x} \\
q_{y} \\
\mp \frac{q^{2}}{q_{z}}
\end{array}\right) .
$$

In the above expressions $q_{z}=\sqrt{1-q^{2}}$ is the normalized $z$ component of the wave vectors and $r_{q}^{\tau}$ is the reflection coefficient for the 2D electron gas. These coefficients are obtained so that the magnetic field $\mathbf{H}$ and electric field $\mathbf{E}$ satisfy the following continuity relations:

$$
\begin{aligned}
\mathbf{e}_{z} \times\left(\mathbf{E}_{+}-\mathbf{E}_{-}\right) & =0, \\
\mathbf{e}_{z} \times\left(\mathbf{H}_{+}-\mathbf{H}_{-}\right) & =\frac{4 \pi}{c} \mathbf{j}=-\frac{4 \pi}{c} \sigma \mathbf{e}_{z} \times\left(\mathbf{e}_{z} \times \mathbf{E}_{+}\right),
\end{aligned}
$$

where $\mathbf{E}_{-}\left(\mathbf{H}_{-}\right)$and $\mathbf{E}_{+}\left(\mathbf{H}_{+}\right)$stay for the electric (magnetic) fields in the regions of negative and positive $z$, respectively, and $\mathbf{e}_{z}$ is the unitary vector along the $+z$ direction. As a result of matching we have

$$
\begin{aligned}
& r_{q}^{\mathrm{TE}}=\frac{-\alpha}{\alpha+q_{z}}, \quad r_{q}^{\mathrm{TM}}=\frac{-\alpha q_{z}}{\alpha q_{z}+1}, \\
& t_{q}^{\mathrm{TE}}=\frac{q_{z}}{\alpha+q_{z}}, \quad t_{q}^{\mathrm{TM}}=\frac{1}{\alpha q_{z}+1},
\end{aligned}
$$

with $\alpha=2 \pi \sigma / c$ being the dimensionless $2 \mathrm{D}$ conductivity.
Explicitly, we have for $\hat{G}_{0}=\hat{G}_{0}^{\mathrm{TM}}+\hat{G}_{0}^{\mathrm{TE}}$

$$
\begin{aligned}
\hat{G}_{0}^{\mathrm{TE}}(\mathbf{r})= & \frac{i k_{\omega}}{8 \pi^{2}} \int \frac{d \mathbf{q}}{q_{z} q^{2}} e^{i \mathbf{q} \mathbf{r}+i q_{z}\left|z-z^{\prime}\right|}\left(\begin{array}{ccc}
q_{y}^{2} & -q_{x} q_{y} & 0 \\
-q_{x} q_{y} & q_{x}^{2} & 0 \\
0 & 0 & 0
\end{array}\right), \\
\hat{G}_{0}^{\mathrm{TM}}(\mathbf{r})= & \frac{i k_{\omega}}{8 \pi^{2}} \int \frac{d \mathbf{q}}{q^{2}} e^{i \mathbf{q r}+i q_{z}\left|z-z^{\prime}\right|} \\
& \times\left(\begin{array}{ccc}
q_{x}^{2} q_{z} & q_{x} q_{y} q_{z} & \mp q_{x} q^{2} \\
q_{x} q_{y} q_{z} & q_{y}^{2} q_{z} & \mp q_{y} q^{2} \\
\mp q_{x} q^{2} & \mp q_{y} q^{2} & q^{4} / q_{z}
\end{array}\right) .
\end{aligned}
$$

Analogously, $\hat{G}_{r}=\hat{G}_{r}^{\mathrm{TM}}+\hat{G}_{r}^{\mathrm{TE}}$ reads

$$
\begin{aligned}
\hat{G}_{r}^{\mathrm{TE}}(\mathbf{r})= & \frac{i k_{\omega}}{8 \pi^{2}} \int \frac{d \mathbf{q}}{q_{z} q^{2}} r_{q}^{\mathrm{TE}} e^{i \mathbf{q r}-i q_{z}\left(z^{\prime}+z\right)}\left(\begin{array}{ccc}
q_{y}^{2} & -q_{x} q_{y} & 0 \\
-q_{x} q_{y} & q_{x}^{2} & 0 \\
0 & 0 & 0
\end{array}\right), \\
\hat{G}_{r}^{\mathrm{TM}}(\mathbf{r})= & \frac{i k_{\omega}}{8 \pi^{2}} \int \frac{d \mathbf{q}}{q^{2}} r_{q}^{\mathrm{TM}} e^{i \mathbf{q r}-i q_{z}\left(z^{\prime}+z\right)} \\
& \times\left(\begin{array}{ccc}
q_{x}^{2} q_{z} & q_{x} q_{y} q_{z} & q_{x} q^{2} \\
q_{x} q_{y} q_{z} & q_{y}^{2} q_{z} & q_{y} q^{2} \\
-q_{x} q^{2} & -q_{y} q^{2} & -q^{4} / q_{z}
\end{array}\right),
\end{aligned}
$$

Finally, for the transmission part $\hat{G}_{t}=\hat{G}_{t}^{\mathrm{TM}}+\hat{G}_{t}^{\mathrm{TE}}$ we have

$$
\begin{aligned}
\hat{G}_{t}^{\mathrm{TE}}(\mathbf{r})= & \frac{i k_{\omega}}{8 \pi^{2}} \int \frac{d \mathbf{q}}{q_{z} q^{2}} t_{q}^{\mathrm{TE}} e^{i \mathbf{q r}+i q_{z}\left(z^{\prime}+z\right)}\left(\begin{array}{ccc}
q_{y}^{2} & -q_{x} q_{y} & 0 \\
-q_{x} q_{y} & q_{x}^{2} & 0 \\
0 & 0 & 0
\end{array}\right), \\
\hat{G}_{t}^{\mathrm{TM}}(\mathbf{r})= & \frac{i k_{\omega}}{8 \pi^{2}} \int \frac{d \mathbf{q}}{q^{2}} t_{q}^{\mathrm{TM}} e^{i \mathbf{q} \mathbf{r}+i q_{z}\left(z^{\prime}+z\right)} \\
& \times\left(\begin{array}{ccc}
q_{x}^{2} q_{z} & q_{x} q_{y} q_{z} & -q_{x} q^{2} \\
q_{x} q_{y} q_{z} & q_{y}^{2} q_{z} & -q_{y} q^{2} \\
-q_{x} q^{2} & -q_{y} q^{2} & q^{4} / q_{z}
\end{array}\right) .
\end{aligned}
$$

\section{APPENDIX C: GREEN'S DYADIC FOR A DIPOLE PLACED AT THE INTERFACE $z=0^{+}$}

We will consider a dipole placed directly onto the graphene sheet, concentrating on the transmission part of the Green's dyadic, $\hat{G}_{t}$. This corresponds to a dipole position slightly below the graphene sheet, i.e., $z^{\prime}=0^{-}$, and to fields created in the region $z>0$. The analytical treatment of the reflection part of the Green's dyadic, $\hat{G}_{r}$, is analogous, and the physical properties of the fields for $z<0$ are similar.

So, supposing that $z^{\prime}=0^{-}$in the previous expressions, the Green's dyadic $\hat{G}(\mathbf{r}, z)=\hat{G}_{t}\left(\mathbf{r}, z, z^{\prime}=0^{-}\right)$can be greatly simplified. The symmetry of the problem makes it convenient to work in cylindrical coordinates $(r, \theta, z)$ (see Fig. 6):

$$
x=r \cos \theta, \quad y=r \sin \theta, \quad z=z .
$$

In this system of coordinates the Green's dyadic can be obtained from the one in Cartesian coordinates through

$$
\hat{G}^{\text {cylindrical }}=\hat{T}^{-1} \hat{G}^{\text {Cartesian }} \hat{T},
$$




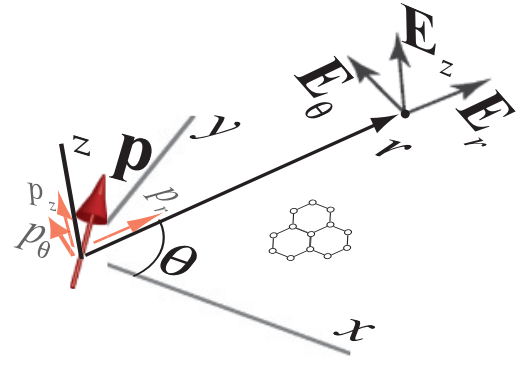

FIG. 6. (Color online) The geometry of the problem: The dipole with the dipole moment $\mathbf{p}$ placed at the origin creates an electric field $\mathbf{E}$ at the point $(r, \theta, 0)$. The plane of the 2DEG coincides with $z=0$. Both Cartesian and cylindrical coordinate systems are shown.

where

$$
\hat{T}=\left(\begin{array}{ccc}
\cos \theta & -\sin \theta & 0 \\
\sin \theta & \cos \theta & 0 \\
0 & 0 & 1
\end{array}\right) .
$$

In polar coordinates the Green's dyadic can be expressed in the form of Sommerfeld integrals involving the angular-spectrum dyadic $\hat{D}(q)$ :

$$
G_{i j}=\frac{i k_{\omega}}{8 \pi} \sum_{\tau=\mathrm{TE}, \mathrm{TM}} \int_{0}^{\infty} d q D_{i j}^{\tau}(q) J_{i j}^{\tau}(q r) e^{i q_{z} z}
$$

with

$$
\begin{aligned}
\hat{J}^{\mathrm{TE}}(q r) & =\left(\begin{array}{ccc}
J_{+}(q r) & 0 & 0 \\
0 & J_{-}(q r) & 0 \\
0 & 0 & 0
\end{array}\right), \\
\hat{J}^{\mathrm{TM}}(q r) & =\left(\begin{array}{ccc}
J_{-}(q r) & 0 & J_{1}(q r) \\
0 & J_{+}(q r) & 0 \\
J_{1}(q r) & 0 & J_{0}(q r)
\end{array}\right) .
\end{aligned}
$$

In these expressions, $J_{ \pm}(q r)=J_{0}(q r) \pm J_{2}(q r)$ and $J_{n}(q r)$ is the Bessel function of $n$th order. The nonzero components of the angular spectrum dyadic are $D_{r r}^{\mathrm{TE}}=D_{\theta \theta}^{\mathrm{TE}}=t_{q}^{\mathrm{TE}} q / q_{z}$, $D_{r r}^{\mathrm{TM}}=D_{\theta \theta}^{\mathrm{TM}}=t_{q}^{\mathrm{TM}} q q_{z}, \quad D_{r z}^{\mathrm{TM}}=D_{z r}^{\mathrm{TM}}=-2 i q^{2} t_{q}^{\mathrm{TM}} / q_{z}$, and $D_{z z}^{\mathrm{TM}}=2 q^{3} t_{q}^{\mathrm{TM}} / q_{z}$.

SPP contribution to the field. The contribution from the SPP is calculated from the residues of the poles in the TM part of the Green's dyadic. For the case of a vertical dipole, this calculation has been performed in Ref. 10. In order to find the SPP field, all Bessel functions must be expressed in terms of Hankel functions of first $\left(H^{(1)}\right)$ and second $\left(H^{(2)}\right)$ kind. Then the contour integral is deformed and closed either in the upper half-plane of complex variable $q$ (for terms involving $H^{(1)}$ ) or in the lower half-plane (terms where $H^{(2)}$ appears). As the SPP pole is enclosed only when closing the integration contour in the half-plane, the final result is

$$
\hat{G}^{\mathrm{SPP}}(r, z)=\frac{k_{\omega}}{8} \frac{q_{p}}{\alpha^{3}} e^{i q_{p z} z}\left(\begin{array}{ccc}
H_{-}^{(1)}\left(q_{p} r\right) & 0 & 2 i q_{p} \alpha H_{1}^{(1)}\left(q_{p} r\right) \\
0 & H_{+}^{(1)}\left(q_{p} r\right) & 0 \\
2 i q_{p} \alpha H_{1}^{(1)}\left(q_{p} r\right) & 0 & 2 q_{p}^{2} \alpha^{2} H_{0}^{(1)}\left(q_{p} r\right)
\end{array}\right),
$$

where $H_{ \pm}^{(1)}(q r)=H_{0}^{(1)}(q r) \pm H_{2}^{(1)}(q r), H_{n}^{(1)}(q r)$ is the firstkind Hankel function of $n$th order, and $q_{p}=\sqrt{1-\alpha^{-2}}$ with $q_{p z}=-\alpha^{-1}$ are the normalized $k$-vector components of the SPP.

Asymptotic expansion of the Green's dyadic at the surface $z=0^{+}$: the leading order. For large values of $r$, such that $r \gg 1$, an asymptotic expansion of the GD can be performed. As in the previous subsection, the limits of the integral (1) are extended to the whole real $q$ axis using the Hankel functions, and then the long-distance asymptotic value of these functions is taken. The result reads

$$
\begin{aligned}
\hat{G}(r, z)= & \frac{k_{\omega} e^{i \frac{\pi}{4}}}{8 \pi} \sqrt{\frac{2}{\pi r}} \int_{-\infty}^{\infty} d q \sqrt{q} e^{i q r+i q_{z} z} \\
& \times\left(\begin{array}{ccc}
\frac{q_{z}}{\alpha q_{z}+1} & 0 & -\frac{q}{\alpha q_{z}+1} \\
0 & \frac{1}{\alpha+q_{z}} & 0 \\
-\frac{q}{\alpha q_{z}+1} & 0 & \frac{q^{2}}{q_{z}\left(\alpha q_{z}+1\right)}
\end{array}\right) .
\end{aligned}
$$

On the plane $z=0^{+}$, the main contribution is expected to proceed from the branch point $q_{z}=0$. Then the computational procedure consists of transforming the integral to a form such that the integrand is proportional to $1 / q_{z}$. The element $z z$ already contains this factor, so the transformation for it is not necessary. The other elements should be integrated by parts. After the transformation, the integrand is expanded in the vicinity of $q_{z}=0$, and then using

$$
\int_{-\infty}^{\infty} d q \frac{e^{i q r}}{q_{z}}=\pi H_{0}^{(1)}(q r)
$$

the integration is trivially performed:

$$
\hat{G}(r, 0)=\frac{k_{\omega} e^{i \frac{\pi}{4}}}{8} \sqrt{\frac{2}{\pi r}}\left(\begin{array}{ccc}
\frac{1}{i r} & 0 & \frac{\alpha}{i r} \\
0 & \frac{i}{r \alpha^{2}} & 0 \\
\frac{\alpha}{i r} & 0 & 1
\end{array}\right) H_{0}^{(1)}(q r) .
$$

Finally, substituting $H_{0}^{(1)}(q r)$ with its asymptotic value, we arrive at

$$
\hat{G}(r, 0)=\frac{k_{\omega}}{4 \pi r}\left(\begin{array}{ccc}
\frac{1}{i r} & 0 & \frac{\alpha}{i r} \\
0 & \frac{i}{r \alpha^{2}} & 0 \\
\frac{\alpha}{i r} & 0 & 1
\end{array}\right) e^{i r} .
$$

The dependency $\sim 1 / r$ is exactly the same as for the case of free space (spherical-type wave in vacuum) while the $\sim 1 / r^{2}$ decay is redolent of the Norton wave arising in radio transmission at the Earth's surface ${ }^{19}$ and of radiation by holes in metals. ${ }^{20}$

Analytical form of the Green's dyadic of free space for $z=0$. The free-space GD follows from Eq. (B2) just by setting $\alpha=0$ so that we simply have $\hat{G}=\hat{G}_{0} . \hat{G}_{0}$ can be 
rearranged as

$$
\hat{G}_{0}(\mathbf{r}, z)=\frac{i k_{\omega}}{8 \pi^{2}} \int \frac{d \mathbf{q}}{q_{z}}\left(\begin{array}{ccc}
1-q_{x}^{2} & -q_{x} q_{y} & -q_{x} q_{z} \\
-q_{x} q_{y} & 1-q_{y}^{2} & -q_{y} q_{z} \\
-q_{x} q_{z} & -q_{y} q_{z} & 1-q_{z}^{2}
\end{array}\right) e^{i \mathbf{q} \mathbf{r}+i q_{z} z}
$$

Using the Green's function for a scalar potential,

$$
G_{0}(\rho)=\frac{k_{\omega}}{4 \pi} \frac{e^{i \rho}}{\rho}, \quad \rho^{2}=r^{2}+z^{2} .
$$

Equation $(\mathrm{C} 11)$ can be written in a differential form:

$$
\hat{G}_{0}(\mathbf{r}, z)=\left(\begin{array}{ccc}
1+\partial_{x}^{2} & \partial_{x} \partial_{y} & \partial_{x} \partial_{z} \\
\partial_{x} \partial_{y} & 1+\partial_{y}^{2} & \partial_{y} \partial_{z} \\
\partial_{x} \partial_{z} & \partial_{y} \partial_{z} & 1+\partial_{z}^{2}
\end{array}\right) G_{0}(\rho)
$$

After performing the derivatives in Eq. (C13), the result in cylindrical coordinates is

$$
\hat{G}_{0}^{\text {cylindrical }}(r, 0)=\frac{k_{\omega}}{4 \pi} \frac{e^{i r}}{r^{3}}\left(\begin{array}{ccc}
2(-1+i r) & 0 & 0 \\
0 & -1+i r+r^{2} & 0 \\
0 & 0 & -1+i r+r^{2}
\end{array}\right) \text {. }
$$

*alexeynik@rambler.ru

†'mm@unizar.es

${ }^{1}$ K. S. Novoselov, A. K. Geim, S. V. Morozov, D. Jiang, M. I. Katsnelson, I. V. Grigorieva, S. V. Dubonos, and A. A. Firsov, Nature (London) 438, 197 (2005).

${ }^{2}$ A. H. Castro Neto, F. Guinea, N. M. R. Peres, K. S. Novoselov, and A. K. Geim, Rev. Mod. Phys. 81, 109 (2009).

${ }^{3}$ A. K. Geim, Science 324, 1530 (2009).

${ }^{4}$ F. Bonaccorso, Z. Sun, T. Hasan, and A. C. Ferrari, Nat. Photonics 4, 611 (2010).

${ }^{5}$ C. Casiraghi, A. Hartschuh, E. Lidorikis, H. Qian, H. Harutyunyan, T. Gokus, K. S. Novoselov, and A. C. Ferrari, Nano Lett. 7, 2711 (2007).

${ }^{6}$ P. Blake, E. W. Hill, A. H. Castro Neto, K. S. Novoselov, D. Jiang, R. Yang, T. J. Booth, and A. K. Geim, Appl. Phys. Lett. 91, 063124 (2007).

${ }^{7}$ Kenneth W.-K. Shung, Phys. Rev. B 34, 979 (1986).

${ }^{8}$ G. Campagnoli and E. Tosatti, in Progress on Electron Properties of Metals, edited by R. Girlanda et al. (Kluwer, Dordrecht, 1989), p. 337.
${ }^{9}$ O. Vafek, Phys. Rev. Lett. 97, 266406 (2006).

${ }^{10}$ G. W. Hanson, J. Appl. Phys. 103, 064302 (2008).

${ }^{11}$ S. A. Mikhailov and K. Ziegler, Phys. Rev. Lett. 99, 016803 (2007).

${ }^{12}$ Yu. V. Bludov, M. I. Vasilevskiy, and N. M. R. Peres, Europhys. Lett. 92, 68001 (2010).

${ }^{13}$ A. Vakil and N. Engheta, Science 332, 1291 (2011).

${ }^{14}$ B. Wunsch, T. Stauber, F. Sols, and F. Guinea, New J. Phys. 8, 318 (2006).

${ }^{15}$ E. H. Hwang and S. Das Sarma, Phys. Rev. B 75, 205418 (2007).

${ }^{16}$ L. A. Falkovsky, Phys. Usp. 51, 887 (2008).

${ }^{17}$ L. Novotny and B. Hetch, Principles of Nano-Optics (Cambridge University Press, New York, 2006).

${ }^{18}$ F. H. L. Koppens, D. E. Chang, and F. J. García de Abajo, Nano Lett. 11, 3370 (2011).

${ }^{19}$ K. A. Norton, Proc. IRE 24, 1367 (1936).

${ }^{20}$ A. Yu. Nikitin, F. J. Garcia-Vidal, and L. Martin-Moreno, Phys. Rev. Lett. 105, 073902 (2010).

${ }^{21}$ L. P. Felsen and N. Marcuvitz, Radiation and Scattering of Waves (IEEE, Piscataway, NJ, 1994). 